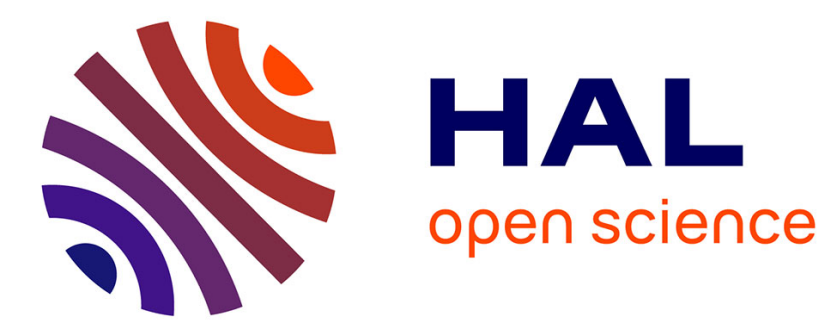

\title{
Le développement de Digitalis lanata (Ehrh.) en conditions contrôlées et naturelles : établissement de critères de sélection
}

Christophe Brugidou, Monique Jacques

\section{To cite this version:}

Christophe Brugidou, Monique Jacques. Le développement de Digitalis lanata (Ehrh.) en conditions contrôlées et naturelles: établissement de critères de sélection. Agronomie, 1987, 7 (9), pp.685-694. hal-00885042

\section{HAL Id: hal-00885042 \\ https://hal.science/hal-00885042}

Submitted on 1 Jan 1987

HAL is a multi-disciplinary open access archive for the deposit and dissemination of scientific research documents, whether they are published or not. The documents may come from teaching and research institutions in France or abroad, or from public or private research centers.
L'archive ouverte pluridisciplinaire HAL, est destinée au dépôt et à la diffusion de documents scientifiques de niveau recherche, publiés ou non, émanant des établissements d'enseignement et de recherche français ou étrangers, des laboratoires publics ou privés. 


\title{
Le développement de Digitalis lanata (Ehrh.) en conditions contrôlées et naturelles : établisse- ment de critères de sélection
}

Christophe BRUGIDOU \& Monique JACQUES

Institut de Physiologie végétale (Phytotron), C.N.R.S., F 91190 Gif-sur-Yvette

\begin{abstract}
La croissance et la floraison de plantes issues de différents lots de graines sont étudiées en conditions contrôlées, afin de définir les conditions de culture les plus favorables à la sélection du Digitalis lanata, ainsi que les critères de cette sélection.

La faculté de former des rosettes axillaires, facteur essentiel d'un bon développement des plantes, peut être un élément discriminateur, élément qui a pu être déterminé grâce à l'emploi de divers types d'éclairements. Tandis qu'un éclairement de $300 \mu \mathrm{Em}^{-2} \mathrm{~s}^{-1}$ ( (Grolux Sylvania ») riche en radiations rouges induit le développement des rosettes axillaires, en jours courts et en jours longs, un éclairement de $250 \mu \mathrm{Em}^{-2} \mathrm{~s}^{-1}$ ( ( Blanc Industrie, Mazda Fluor ") moins riche en radiations photosynthétiquement actives n'est stimulateur qu'en jours longs. La photopériode de $9 \mathrm{~h}$ et $250 \mu \mathrm{Em}^{-2} \mathrm{~s}^{-1}$ permet de déceler des plantes peu exigeantes vis-à-vis de la photopériode pour le développement des axillaires alors que l'éclairement de $300 \mu \mathrm{Em}^{-2} \mathrm{~s}^{-1}$ en $9 \mathrm{~h}$ révèle les plantes les plus précoces pour ce développement.

Les plantes au stade 30-35 feuilles fleurissent en jours longs à $22^{\circ} \mathrm{C}$ après avoir subi 7 semaines de froid. Pour toutes les plantes expérimentées les jours longs sont indispensables à l'expression florale. L'étude de l'induction florale par le froid a révélé des sensibilités différentes entrę les plantes (intra- et inter-lots) pour la floraison. Quelques plantes peuvent fleurir sans froid si l'éclairement est suffisamment intense $\left(16 \mathrm{~h}, 300 \mu \mathrm{Em}^{-2} \mathrm{~s}^{-1}\right.$, "Grolux Sylvania »). Enfin sur le terrain la défoliation pratiquée sur des plantes jeunes permet de distinguer les individus par leurs degrés de propension à la floraison.
\end{abstract}

Mots clés additionnels : Développement végétatif, dominance apicale, floraison, vernalisation, défoliation. criteria for selection.

Growth and flowering of plants from various seed samples were studied under controlled conditions to define the most adequate selection conditions and selection criteria for foxglove (Digitalis lanata).

The aptitude to develop axillary rosettes was an essential factor for satisfactory development of the plants. This discriminatory element could be detected and tested by means of a variety of types of light conditions. While $300 \mu \mathrm{Em}^{-2} \mathrm{~S}^{-1}$ ("Grolux Sylvania") lighting rich in red radiation led to development of axillary rosettes during short days as well as long days, $250 \mu \mathrm{Em}^{-2} \mathrm{~s}^{-1}$ ("Blanc Industrie, Mazda Fluor"), less rich in photosynthetically active radiation, produced a stimulatory effect only during long days. A $9 \mathrm{~h}$ photoperiod at $250 \mu \mathrm{Em}^{-2} \mathrm{~s}+1$ was enough for plants with limited photoperiodic requirements to develop axillary rosettes, while $9 \mathrm{~h}$ a1 $300 \mu \mathrm{Em}^{-2} \mathrm{~s}^{-1}$ discriminated the most precocious plants and the best adapted to axillary development.

At the 30-35 leaf stage, plants flowered on long days at a temperature of $22{ }^{\circ} \mathrm{C}$ after having undergone seven weeks of cold conditions. All experimental plants needed long days to flower. The study of cold-induced flowering revealed a differential perceptiveness among plants, according to whether they came from intra- or from inter-samplings. A limited number of plants flowered without cold treatment if light-conditions were satisfactorily intense (16 h at $300 \mu \mathrm{Em}^{-2} \mathrm{~s}^{-1}$ "Grolux Sylvania"). Field defoliation treatments on young plants brought out differential features according to degrees of propensity to flowering.

Additional key words : Vegetative development, apical dominance, flowering, vernalization, defoliation.

\section{INTRODUCTION}

La digitale laineuse (Digitalis lanata (Ehrh.), famille des Scrofulariacées) est une plante bisannuelle allogame. Elle produit à la fin de la première année de végétation des hétérosides cardiotoniques, extraits des feuilles séchées (PARIS \& MOYSE, 1971; HOLLMAN, 1985). Actuellement, la digoxine est dans le monde le premier hétéroside cardiotonique utilisé pour traiter l'insuffisance cardiaque et les arythmies supraventriculaires. 
La Hollande ayant entrepris la sélection de la digitale laineuse dès 1972 (MASTENBROEK, 1979 et 1985) est maintenant le principal producteur mondial. Depuis 1968 la production française traditionnellement implantée dans le Maine-et-Loire (HERRISSET, 1968), n'a pu faire face à partir des années 80 à la concurrence hollandaise. Depuis cette époque, quelques travaux ont été entrepris sur la biologie et l'amélioration de cette plante : culture et sélection (LOISELEUR, 1980), multiplication végétative in vitro (LÉVY, 1981), et biologie florale (PASQUIER, 1983). L'étude du développement végétatif et floral ayant eu lieu en conditions non contrôlées n'a pas conduit à une connaissance précise du cycle biologique, de même, des caractères précoces de croissance, impliqués sur le rendement en matière sèche n'ont pu être dégagés. Le travail de LoISELEUR (1980) a permis de sélectionner en fin de culture 4 lots de plantes uniquement sur des critères de rendement de matière sèche et de teneur en digoxine.

L'objectif de ce travail est donc de répondre rapidement à l'attente des producteurs et des industriels, il a été engagé dans le but d'amener la production nationale sur le plan quantitatif et qualitatif à un niveau compétitif satisfaisant. C'est à partir de ces 4 lots de graines que notre travail s'est orienté vers la recherche de méthodes de sélection à des stades de développement précoces, pour augmenter le rendement de matière sèche à l'hectare et pour améliorer la pratique culturale de cette plante. Nous relatons ici, les résultats d'une étude écophysiologique sur la croissance et la floraison de la digitale laineuse en conditions contrôlées et en conditions naturelles. A notre connaissance ce sont les seuls résultats décrivant précisément le cycle biologique de la digitale laineuse. L'étude de la floraison s'appuiera d'une part, sur les principes classiques d'étude de la vernalisation, définie comme un processus préparatoire à la floraison (LANG, 1965 ; PICARD, 1968 ; CHOUARD, 1969) et d'autre part, sur les travaux réalisés sur la digitale pourpre, espèce voisine de la digitale laineuse où la vernalisation a été particulièrement étudiée (PICARD, 1966 ; PHELOUZAT \& PICARD, 1968 ; PHElOUZAT, 1970). Le processus de mise à fleurs mettant en jeu des corrélations entre les différents organes de la plante (MIGINIAC, 1978), nous avons entrepris en plus une expérimentation en pratiquant des ruptures de corrélations par défoliation. Sur les 5 lots de plantes disponibles, l'observation précise de la croissance et de la floraison a révélé une grande variabilité. Nous avons pu définir ainsi des conditions de culture capables de révéler un certain nombre de potentialités de variabilité intra- et inter-lots et dégager quelques éléments de sélection pour repérer des plantes dont le processus de croissance et de floraison satisfasse aux exigences des producteurs : tout d'abord des plantes présentant une bonne croissance et ensuite des plantes étant des bisanuelles strictes pour leur floraison.

\section{MATÉRIEL ET MÉTHODES}

\section{A. Le matériel végétal}

Le matériel végétal est obtenu à partir, d'une part :

- des 4 lots de graines P7, P9, L3-2 et L4-2 fournies par l'Institut Technique des Plantes Médicinales Aro- matiques et Industrielles (ITEPMAI), issues de plantes sélectionnées selon des critères morphologiques et biochimiques (LOISELEUR, 1980) ;

- d'autre part, d'un lot de graines noté L3-2 1A, obtenues par autofécondation d'une plante du lot L32, sélectionnée en 1984 par nos soins sur un critère de teneur en digoxine.

\section{B. Conditions de culture}

Les semis sont effectués sur vermiculite ; 3 semaines plus tard les plantes (stade 2 feuilles) sont repiquées en pot de $8 \mathrm{~cm}$ de diamètre, puis cultivées selon les conditions décrites ci-dessous.

Les expériences à Gif-sur-Yvette se déroulent soit en conditions contrôlées (serre à éclairement artificiel complémentaire de l'éclairement naturel et enceintes climatisées), soit en milieu naturel.

En conditions contrôlées, les plantes sont cultivées sur vermiculite à une température de $22^{\circ} \mathrm{C}$ et $60 \mathrm{p} .100$ d'humidité relative et arrosées 2 fois par jour : solution nutritive (mise au point par NITSCH, décrite par DE BILDERLING \& LOURTIOUX, 1976) le matin, eau déminéralisée le soir. En serre l'énergie de l'éclairement varie au cours de l'année mais, grâce à un éclairement complémentaire, n'est jamais inférieur à $125 \mu \mathrm{Em}^{-2} \mathrm{~s}^{-1}$ («Blanc Industrie, Mazda Fluor» de $40 \mathrm{~W}$ et lampes incandescentes de $75 \mathrm{~W})$. En enceintes climatisées, l'éclairement artificiel est soit d'environ $250 \mu \mathrm{Em}^{-2} \mathrm{~s}^{-1}$ («Blanc Industrie, Mazda Fluor» de $65 \mathrm{~W}$ et lampes incandescentes de $15 \mathrm{~W}$ ) soit d'environ $300 \mu \mathrm{Em}^{-2} \mathrm{~S}^{-1}$ («Grolux Sylvania » : tubes Grolux W. S. Sylvania de $215 \mathrm{~W})$. Ces types d'éclairements diffèrent donc par leur flux énergétique mais aussi par leur qualité spectrale, les tubes «Grolux » étant environ 10 fois plus riches en radiations rouge clair que les tubes Blanc Industrie.

En milieu naturel, la pratique culturale adoptée est celle du Maine-et-Loire (LOISELEUR, 1980) ; les plantes des lots P7 et L3-2 1A, préalablement cultivées en serre sur du terreau, sont repiquées le 10-05-85 au stade 510 feuilles en pleine terre. Il n'y a ni irrigation, ni apport d'engrais pendant la durée de la culture qui a eu lieu de mai à octobre 85 .

\section{Conditions de vernalisation}

Les plantes $\mathrm{P} 7$ et $\mathrm{P} 9$ préalablement cultivées en conditions contrôlées sous une photopériode de $9 \mathrm{~h}$, sont, au stade morphologique choisi, réparties en lots de 5 plantes. Elles sont soumises à la vernalisation pendant des durées variées, sous un éclairement artificiel de $250 \mu 1 \mathrm{~m}^{-2} \mathrm{~s}^{-1}$ (« Mazda fluor $\left.»\right)$, à une température de $5{ }^{\circ} \mathrm{C}$ et une photopériode de $9 \mathrm{~h}$. La réponse florale est observée après le retour des plantes à $22^{\circ} \mathrm{C}$ en serre de février à mai, soit en $9 \mathrm{~h}$, soit en $16 \mathrm{~h}$.

\section{Critères d'observation de la floraison}

La réponse au traitement inducteur de la floraison est appréciée selon différents critères :

- la durée minimale du traitement inducteur nécessaire pour amener une floraison minimale (floraison de la hampe principale issue de la rosette centrale) ;

- la proportion de plantes fleuries ; 
- la durée de l'expression florale (temps écoulé entre la fin du traitement inducteur et le début de la montaison) ; les).

- l'intensité de floraison (nombre de hampes flora-

\section{RÉSULTATS}

\section{A. Croissance végétative}

1. Croissance sous un éclairement de $300 \mu \mathrm{Em}^{-2} \mathrm{~S}^{-1}$ ("Grolux Sylvania »)

Les feuilles nouvellement dégagées d'une longueur de $1 \mathrm{~cm}$ sont comptées toutes les semaines sur les plantes des lots P7, P9, L3-2, L4-2. Les cinétiques de croissance des 4 lots sont regroupées 2 par 2, les lots P7 et P9 ainsi que les lots L3-2 et L4-2 se comportant de la même façon (fig. 1). La courbe représentant la vitesse

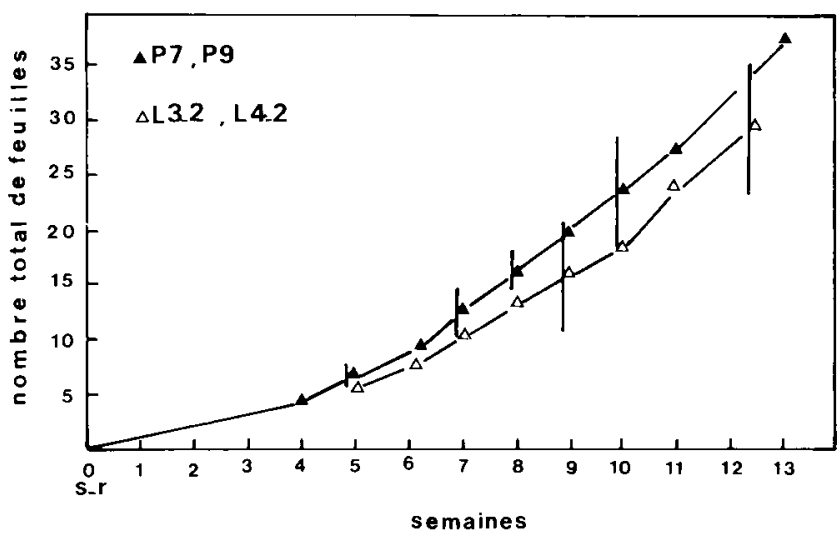

Figure 1

Croissance de la rosette principale de 4 lots de Digitalis lanata. Eclairement: $S \mathrm{~h}, 300 \mu \mathrm{Em}^{-2} \mathrm{~s}^{-1}$; Semis-Repiquage (S.R.): 3 semaines. Chaque point : moyenne des valeurs de 6 plantes. Les traits verticaux représentent l'écart-type.

Growth of main rosette for four samples of Digitalis lanata. Light condition : $9 \mathrm{~h}, 300 \mu \mathrm{Em}^{-2} \mathrm{~s}^{-1}$; time from sowing to pricking out (S.R.).

Each point is the average of six measurements. Vertical bars represent standard deviation.

de dégagement des feuilles est assimilée à une succession de droites correspondant à des intervalles de temps déterminés.

La croissance est lente (pente de 0,3 ) jusqu'à la $5^{\mathrm{e}}$ semaine après le repiquage; au-delà le nombre de feuilles augmente rapidement : la pente de 0,7 entre la $7^{\mathrm{e}}$ et la $10^{\mathrm{e}}$ semaine passe à 0,95 après la $10^{\mathrm{e}}$ semaine. Pour de jeunes plantes, il n'y a pas de différence marquée entre les 4 lots de digitale pour la croissance de la rosette initiale.

Après 7 semaines de culture, en $9 \mathrm{~h}$ et $16 \mathrm{~h}$ d'éclairement, il apparaît en moyenne pour les lots P7 et P9 4 feuilles par semaine (fig. 2). A la $8^{\mathrm{e}}$ semaine, un décrochement est observé : le nombre de feuilles apparues passe de 4 à 2,5, il correspond à la mise en place des rosettes axillaires. Pour un éclairement de $300 \mu \mathrm{Em}^{-2} \mathrm{~s}^{-1}$, et quelle que soit la photopériode, les rosettes axillaires sont visibles à partir de la $8^{e}$ semaine. Après le débourrement des rosettes axillaires, le rythme d'apparition des feuilles de la rosette centrale aug-

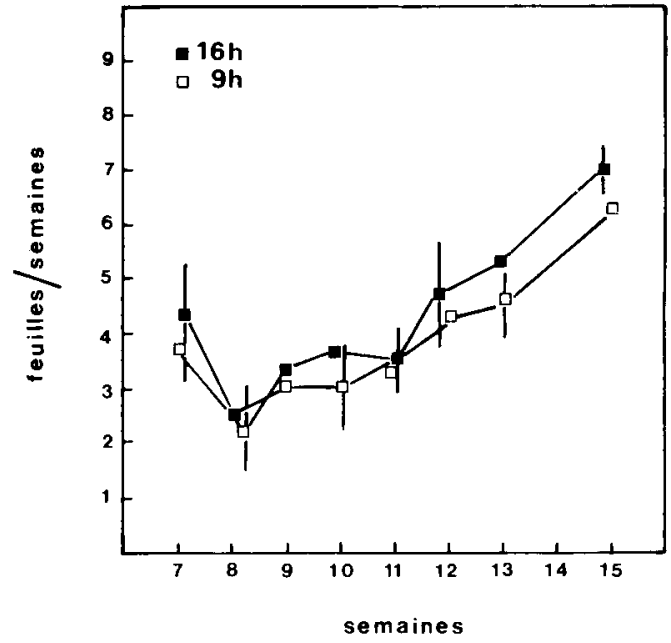

Figure 2

Influence de la photopériode sur la vitesse de dégagement des feuilles des lots $P 7$ et $P 9$ de Digitalis lanata.

Eclairement : $9 \mathrm{~h}, 300 \mu \mathrm{Em}^{-2} \mathrm{~s}^{-1}$.

Chaque point : moyenne des valeurs de 6 plantes. Les traits verticaux représentent l'écart-type.

Influence of photoperiod on rate of leaf emergence : samples $P 7$ and $P 9$ of Digitalis Lanata.

Light condition : $9 \mathrm{~h}, 300 \mu \mathrm{Em}^{-2} \mathrm{~s}^{-1}$.

Each point is the average of six measurements. Vertical bars represent standard deviation.

mente de la même façon pour les 2 photopériodes. Toutefois, la photopériode de $16 \mathrm{~h}$ reste la plus favorable sur l'activité foliaire de la rosette centrale et sur la vitesse de développement des rosettes axillaires. Le rythme d'apparition est d'environ une feuille par jour à la $15^{\mathrm{e}}$ semaine de culture, au-delà, la densité foliaire de la plante est telle que l'étude du rythme de formation des feuilles sur la rosette centrale devient impossible.

Cette étude, sous un éclairement de $300 \mu \mathrm{Em}^{-2} \mathrm{~s}^{-1}$, donne lieu à 2 remarques :

- l'utilisation d'un éclairement de $300 \mu \mathrm{Em}^{-2} \mathrm{~s}^{-1}$ qui provoque le développement des rosettes axillaires à la fois en $9 \mathrm{~h}$ et $16 \mathrm{~h}$ a pu masquer un effet photopériodique ;

- indépendamment de la photopériode, il semble exister une variabilité entre les plantes d'un même lot pour l'apparition des rosettes axillaires.

Par la suite, nous nous sommes donc attachés à définir grâce à un autre éclairement $\left(250 \mu \mathrm{Em}^{-2} \mathrm{~s}^{-1}\right.$ "Mazda Fluor ») le rôle de la photopériode sur le développement des rosettes axillaires et à différencier en conditions semi-optimales de développement (photopériode $9 \mathrm{~h}$, éclairement $300 \mu \mathrm{Em}^{-2} \mathrm{~S}^{-1}$ « Grolux Sylvania $»)$ les 4 lots disponibles par leur aptitude au développement des rosettes axillaires.

\section{Croissance sous un éclairement de $250 \mu \mathrm{Em}^{-2} \mathrm{~S}^{-1}$ "Blanc Industrie, Mazda Fluor »}

A partir du mois de septembre 1985 , après 10 semaines de culture en serre sous une photopériode de $9 \mathrm{~h}$, les plantes du lot L3-2 1A ont atteint des stades morphologiques variés. Les plantes d'une vingtaine de feuilles sont soumises à l'expérimentation. Ces plantes ne portent pas de rosette axillaire au moment de leur 
transfert en $9 \mathrm{~h}$ et $16 \mathrm{~h}$, en conditions phytotroniques strictes.

Lorsque l'éclairement est de $250 \mu \mathrm{Em}^{-2} \mathrm{~S}^{-1}$, seule une photopériode de $16 \mathrm{~h}$ stimule le développement des rosettes axillaires (fig. $3 \mathrm{a}^{\prime}$ ). En une semaine et demie, toutes les plantes possèdent au moins 10 rosettes axillaires. Parallèlement à la mise en place des rosettes axillaires une baisse d'activité du méristème central est observée (fig. 3a), en 2 semaines le nombre moyen de feuilles apparues par semaine passe de 6 à 3,5 .
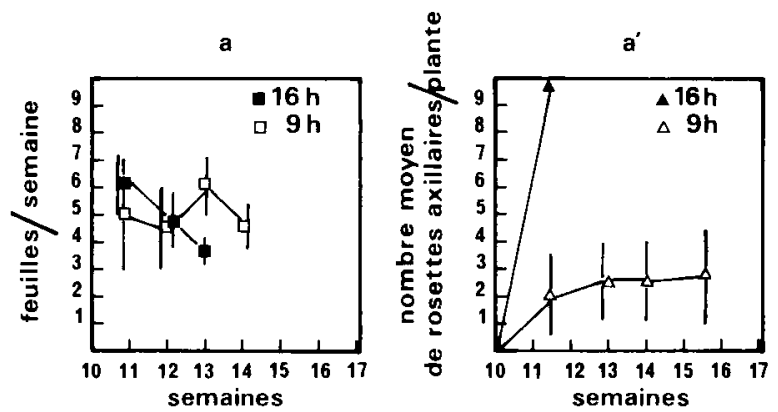

Figure 3

Influence de la photopériode sur le dégagement des feuilles et le développement des rosettes axillaires des plantes $L 3-21 A$ d'une vingtaine de feuilles.

Eclairement : $250 \mu \mathrm{Em}^{-2} \mathrm{~s}^{-1}$

Chaque point : moyenne des valeurs de 5 plantes. Les traits verticaux représentent l'écart-type.

Influence of photoperiod on leaf emergence and axillary rosette development of plants $L 3-21 \mathrm{~A}$ with about twenty leaves.

Light condition : $250 \mu \mathrm{Em}^{-2} \mathrm{~s}^{-1}$.

Each point is the average of five measurements. Vertical bars represent standard deviation.

Une photopériode de $9 \mathrm{~h}$ est défavorable à la formation des rosettes axillaires car, il n'y a pas plus de 3 rosettes axillaires formées (fig. $3 \mathrm{a}^{\prime}$ ). L'activité du méristème central reste alors à peu près stable avec 5 feuilles formées par semaine (fig. 3a). Toutefois dans ces conditions, nous pouvons observer quelques plantes, qui tout en gardant un rythme constant d'apparition de feuilles, se distinguent par un développement des rosettes axillaires.

Un éclairement de $250 \mu \mathrm{Em}^{-2} \mathrm{~S}^{-1}$ a révélé un effet de la photopériode sur le rythme de dégagement des feuilles et sur le développement des rosettes axillaires. Il est moins favorable à la croissance qu'un éclairement de $300 \mu \mathrm{Em}^{-2} \mathrm{~s}^{-1}$, celui-ci assurant le développement de la rosette centrale et des rosettes axillaires.

\section{Influence du stade de croissance sur le développe- ment des rosettes axillaires}

Le développement des plantes L3-2 1A d'une dizaine de feuilles est comparé à celui des plantes d'une vingtaine de feuilles en $9 \mathrm{~h}$ et $16 \mathrm{~h}$. Comme nous avons pu l'observer sur des plantes possédant une vingtaine de feuilles (fig. $3 \mathrm{a}$ et $3 \mathrm{a}^{\prime}$ ), une photopériode de $16 \mathrm{~h}$ stimule le développement des rosettes axillaires lorsque les plantes ne comptent qu'une dizaine de feuilles (fig. $4 \mathrm{~b}$ et $4 \mathrm{~b}^{\prime}$ ). Le temps nécessaire pour que toutes les plantes possèdent 10 rosettes axillaires est alors de 3 semaines. Par contre, sur la rosette centrale le rythme d'apparition des feuilles est constant ( 3 à 4 feuilles par semaine) et reste au même niveau que celui d'une photopériode de $9 \mathrm{~h}$ (fig. 4b). Lorsque les plantes ont une dizaine de feuilles, l'effet défavorable d'une photopériode de $9 \mathrm{~h}$ sur le développement des rosettes axillaires est accentué, une seule rosette axillaire par plante étant observée (fig. $4 b^{\prime}$ ).
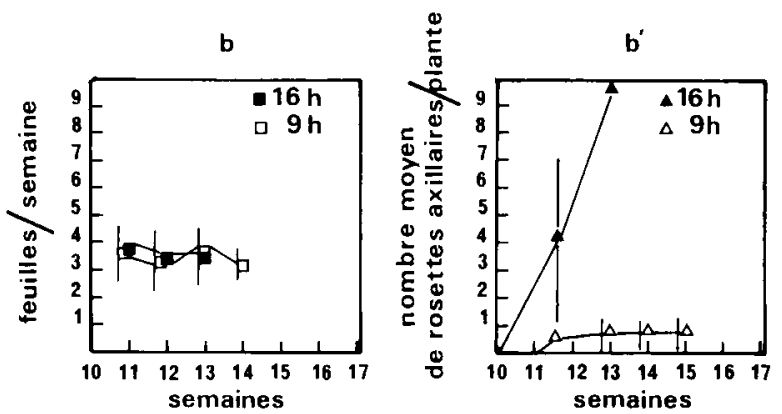

Figure 4

Influence de la photopériode sur le dégagement des feuilles et le développement des rosettes axillaires des plantes L3-2 1A d'une dizaine de feuilles.

Eclairement : $250 \mu \mathrm{Em}^{-2} \mathrm{~s}^{-1}$.

Chaque point : moyenne des valeurs de 5 plantes. Les traits verticaux représentent l'écart-type.

Influence of photoperiod on leaf emergence and axillary rosette development of plants L3-2 $1 \mathrm{~A}$ with about ten leaves.

Light condition : $250 \mu \mathrm{Em}^{-2} \mathrm{~s}^{-1}$.

Each point is the average of five measurements. Vertical bars represent standard deviation.

4. Mise en évidence d'une variabilité entre les plantes sur la vitesse de développement des rosettes axillaires en conditions semi-optimales

A partir des plantes P7, P9, L3-2 et L4-2, des lots de 20 plantes sont constitués et placés sous un éclairement de $300 \mu \mathrm{Em}^{-2} \mathrm{~S}^{-1}$ en $9 \mathrm{~h}$. Le nombre de plantes ayant formé des rosettes axillaires est noté en fonction du temps et ceci quel que soit le nombre de rosettes formées. Entre la $9^{\mathrm{e}}$ semaine et la $14^{\mathrm{e}}$ semaine de culture, le nombre de plantes présentant des rosettes axillaires augmente (fig. 5). L'homogénéité du lot P9 se manifeste par l'apparition de rosettes axillaires dès la $11^{\mathrm{e}}$ semaine de culture chez toutes les plantes. Pour les autres lots, cette apparition est plus progressive, seul le lot P7 se distingue par son hétérogénéité, quelques plantes ne présentent aucune rosette axillaire après 14 semaines de culture. Un éclairement de $300 \mu \mathrm{E}^{-2} \mathrm{~S}^{-1}$ associé à une photopériode courte permet, outre la for-

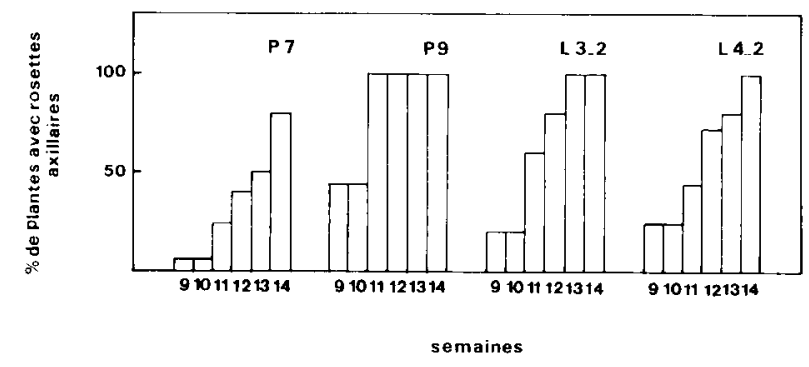

Figure 5

Apparition des premières rosettes axillaires des plantes $P 7, P 9, L 3-2$ et $L 4-2$.

Condition de croissance : $9 \mathrm{~h}, 22^{\circ} \mathrm{C}, 300 \mu \mathrm{Em}^{-2} \mathrm{~s}^{-1}$.

First axillary rosette appearance on $P 7, P 9, L 3-2$ and $L 4-2$ plants. Growth conditions : $9 \mathrm{~h}, 22{ }^{\circ} \mathrm{C}, 300 \mu \mathrm{Em}^{-2} \mathrm{~s}^{-1}$. 
mation de rosettes axillaires, de révéler une variabilité inter- et intra-lot liée au développement de ces dernières.

\section{B. La floraison}

1. Recherche de la durée minimale de froid nécessaire à la floraison en fonction de l'âge des plantes

Les plantes des lots P7 et P9 ayant atteint des stades morphologiques divers sont soumises à la vernalisation.
Durant cette étude, des floraisons se sont produites rapidement après le froid ( 20 à 30 jours) ; elles sont à notre avis les seules réponses spécifiques à l'action du froid vernalisant (chiffres non soulignés tabl. 1 et 2). En revanche, les floraisons qui se sont produites 3 mois après la fin du froid ne nous semblent pas une conséquence directe de l'action du froid (chiffres soulignés). Pour ces 2 lots quel que soit le stade de développement aucune floraison n'apparaît après moins de 8 semaines de traitement par le froid (tabl. 1 et 2). Au-delà, la réponse florale augmente avec la durée de froid et le

TABLEAU 1

Caractéristiques de la floraison des plantes du lot P9 vernalisées à différents stades de développement et en fonction de la durée de froid. Conditions de culture : - Prévernalisation, $9 \mathrm{~h}, 22{ }^{\circ} \mathrm{C}, 300 \mu \mathrm{Em}^{-2} \mathrm{~s}^{-1} ;-$ Vernalisation, $9 \mathrm{~h}, 5^{\circ} \mathrm{C}, 250 \mu \mathrm{Em}{ }^{-2} \mathrm{~s}^{-1} ;-\mathrm{Post}_{-\mathrm{vernalisation}}$ $16 \mathrm{~h}, 22{ }^{\circ} \mathrm{C}$, éclairage naturel (début de l'expérience Mars 1984).

$a$ b c : - $a:$ nombre de plantes en montaison ; $-b:$ délai en jours entre la fin du froid et le début de la montaison; - $c:$ i hampe principale, ++ hampe principale $+2 \grave{a} 3$ hampes axillaires, +++ hampe principale +4 à 5 hampes axillaires.

Nombre de plantes par condition : 5.

Flowering characteristics of plants of sample $P Q$ vernalized at different developrnental stages according to time of cold treatment. Growth conditions: - before vernalization, $9 \mathrm{~h}, 22^{\circ} \mathrm{C}, 300 \mu \mathrm{Em}^{-2} \mathrm{~s}^{-1} ;$ - vernalization, $9 \mathrm{~h}, 5^{\circ} \mathrm{C}, 250 \mu \mathrm{Em}{ }^{-2} \mathrm{~s}^{-1} ;-$ after vernalization, $16 \mathrm{~h}, 22{ }^{\circ} \mathrm{C}$, natural light (start of experiment March 1984).

$a b c:-a:$ number of bolting plants ; $-b:$ time in days between end of cold treatment and beginning of bolting ; $-c:$ inain stem, ++ main stem +2 at 3 axillary stems, +++ main stem +4 at 5 axillary stems.

Number of plants per condition : 5 .

\begin{tabular}{|c|c|c|c|c|c|c|c|c|c|c|c|}
\hline & $\begin{array}{l}\text { Temps de froid } \\
\text { en semaines }\end{array}$ & 4 & & 6 & & & 8 & & & 10 & \\
\hline $\begin{array}{l}\text { Stades morphologiques } \\
5-10 \text { feuilles }\end{array}$ & & $\begin{array}{l}\mathrm{a} \\
0\end{array}$ & $\begin{array}{l}a \\
0\end{array}$ & $\mathrm{~b}$ & $\mathrm{c}$ & $\begin{array}{l}\mathrm{a} \\
0\end{array}$ & $\mathrm{~b}$ & $\mathrm{c}$ & $\begin{array}{l}a \\
0\end{array}$ & $b$ & $c$ \\
\hline 10-15 feuilles & & 0 & 1 & $(\underline{210})$ & + & 0 & & & 0 & & \\
\hline $15-20$ feuilles & & 0 & 0 & & & 1 & $(124)$ & + & 2 & $\begin{array}{l}(30) \\
(30)\end{array}$ & $\begin{array}{l}+ \\
+\end{array}$ \\
\hline 20-25 feuilles & & 0 & 0 & & & 1 & (20) & + & 5 & $\begin{array}{l}(10) \\
(10) \\
(20) \\
(20) \\
(20)\end{array}$ & $\begin{array}{r}++ \\
+++ \\
++ \\
++ \\
++\end{array}$ \\
\hline
\end{tabular}

TABLEAU 2

Caractéristiques de la floraison des plantes du lot P7 vernalisées à différents stades de développement et en fonction de la durée de froid Conditions de culture et expression des résultats (voir tabl. 1). Nombre de plantes par condition : 5 .

Flowering characteristics of plants of sample $P 7$ vernalized at different development stages according to time of cold treatment. Growth conditions and expression of results as in table 1. Number of plants per condition : 5 .

\begin{tabular}{|c|c|c|c|c|c|c|c|c|c|c|c|c|c|}
\hline & $\begin{array}{l}\text { Temps de froid } \\
\text { en semaines }\end{array}$ & & 4 & & & 6 & & & 8 & & & 10 & \\
\hline $\begin{array}{l}\text { Stades morphologiques } \\
5-10 \text { feuilles }\end{array}$ & & 0 & $\mathrm{~b}$ & $\mathrm{c}$ & 0 & b & c & 0 & $\mathrm{~b}$ & $c$ & 4 & $\begin{array}{c}\mathrm{b} \\
(118) \\
(177) \\
(163) \\
(180)\end{array}$ & $\begin{array}{l}c \\
+ \\
+ \\
+ \\
+\end{array}$ \\
\hline $10-15$ feuilles & & 2 & $\begin{array}{l}(94) \\
(150)\end{array}$ & $\begin{array}{l}+ \\
+\end{array}$ & 2 & $\begin{array}{l}(130) \\
(210)\end{array}$ & $\begin{array}{l}+ \\
+\end{array}$ & 2 & $\begin{array}{l}(1.35) \\
(17)\end{array}$ & $\begin{array}{l}+ \\
+\end{array}$ & 2 & $\begin{array}{l}\text { (25) } \\
\text { (25) }\end{array}$ & $\begin{array}{l}+ \\
+\end{array}$ \\
\hline 15-20 feuilles & & 0 & & & 0 & & & 2 & $\begin{array}{l}(45) \\
(180)\end{array}$ & $\begin{array}{l}+ \\
+\end{array}$ & 4 & $\begin{array}{r}(15) \\
(20) \\
(30) \\
(160)\end{array}$ & $\begin{array}{l}+ \\
+ \\
+ \\
+\end{array}$ \\
\hline $20-25$ feuilles & & 1 & (83) & + & 0 & & & 2 & $\begin{array}{l}(20) \\
(15) \\
(15)\end{array}$ & $\begin{array}{c}++ \\
+ \\
+\end{array}$ & 5 & $\begin{array}{r}(20) \\
(20) \\
(90) \\
(125)\end{array}$ & $\begin{array}{c}+++ \\
++ \\
+ \\
+\end{array}$ \\
\hline
\end{tabular}


stade de développement. L'effet synergique de ces 2 facteurs est très net pour le lot $P 9$, au stade 20 25 feuilles après 10 semaines de froid 5 plantes fleurissent, alors qu'après 8 semaines une seule plante avait fleuri (tabl. 1). Au stade 30-35 feuilles, une étude similaire a été réalisée pour les lots L3-2 et L4-2. Une durée de froid de 7 semaines est suffisante pour faire fleurir toutes les plantes, le délai de montaison est de 15 jours. Ainsi, en choisissant un stade de développement plus tardif au moment du traitement vernalisant la durée de froid peut être réduite et une réponse simultanée de la montaison de la rosette centrale et des rosettes axillaires en un temps plus court (15 jours) peut être observée.

Ce type d'étude a permis de révéler, au niveau de la réponse florale, une variabilité inter- et intra-lots. Le lot P7 (tabl. 2) est sensible au froid à un stade plus précoce que le lot $\mathrm{P} 9$. Les premières floraisons du lot $\mathrm{P} 7$ sont observées après 8 semaines de froid ( 2 au stade 10 15 feuilles, 2 au stade 20-25 feuilles) avec un délai de montaison d'une vingtaine de jours, tandis que le lot P9 ne présente qu'une seule floraison au stade 20 25 feuilles avec le même délai de montaison. De plus, les floraisons non liées à l'action du froid sont beaucoup plus nombreuses pour le lot P7 (13 sur 60) que pour le lot P9 (2 sur 60). Enfin, l'observation des lots témoins, non soumis à l'action du froid, confirme ces caractéristiques, puisque, pour le lot P7 2 plantes sur 5 fleurissent, alors qu'aucune ne fleurit pour le lot P9. Ces sensibilités différentes expliquent les montaisons précoces en conditions naturelles.

Il nous a semblé nécessaire de rechercher d'autres facteurs inducteurs de la floraison. L'éclairement a été choisi car c'est le seul facteur majeur pouvant expliquer les montaisons observées en serre au tiède pour les lots $\mathrm{P} 7$ et $\mathrm{P} 9$.

\section{Influence de la photopériode sur l'expression florale}

Aucune floraison n'est observée pour les lots P7 et P9 en 9 h d'éclairement. En 16 h, le nombre de plantes en fleurs dépend du stade morphologique et de la durée de la vernalisation (cf. paragraphe précédent).

La digitale laineuse est donc, pour son expression florale, une plante de jours longs.

\section{Influence de la valeur de l'éclairement sur l'expres- sion florale}

Des plantes du lot L3-2 1A âgées de 4 mois (stade supérieur au stade 30-35 feuilles) subissent, après avoir été cultivées en enceintes climatisées en $9 \mathrm{~h}$ d'éclairement, des durées variables de froid. L'expression florale a lieu en $16 \mathrm{~h}$, soit en éclairement artificiel de $300 \mu \mathrm{Em}^{-2} \mathrm{~s}^{-1}$, soit en éclairement naturel moins intense qui est en moyenne de $160 \mu \mathrm{Em}^{-2} \mathrm{~s}^{-1} \mathrm{de}$ décembre à mars.

Sans froid, un éclairement artificiel intense de $300 \mu \mathrm{Em}^{-2} \mathrm{~s}^{-1}$ suffit à assurer la floraison de quelques plantes (2 sur 5) (tabl. 3). Dans ce cas, seule la hampe principale se développe. Après 5 semaines de froid la proportion de plantes en montaison reste la même, mais 3 hampes axillaires apparaissent 1 semaine après le début de montaison de la hampe principale, celles-ci se développant 15 à 20 jours après la sortie du froid.

En éclairement naturel, ce n'est qu'après 6 semaines de froid qu'une plante développe sa hampe principale 25 jours après la réfrigération. De plus, cette plante ne porte pas de hampes axillaires.

Par contre, sous un éclairement de $300 \mu \mathrm{Em}^{-2} \mathrm{~s}^{-1}$ pour une même durée de froid, nous observons un effet synergique froid-éclairement. En effet, 15 jours après la réfrigération, 5 montaisons sont observées avec une moyenne de 7 hampes axillaires, leur développement étant simultané à celui de la hampe principale. Une augmentation de la durée de froid amplifie l'effet synergique : 8 à 11 hampes axillaires se développent de façon synchrone avec la hampe principale.

En conditions naturelles pour les mêmes durées de froid, on observe 100 p. 100 de montaison, mais l'intensité de la floraison est réduite (tabl. 3). Les hampes axillaires, moins nombreuses montent après la hampe principale, elles sont chétives et le nombre de fleurs qu'elles portent est réduit.

\section{Rôle des feuilles dans la mise à fleurs}

Au cours du temps des plantes des lots P7 et L3-2 13A sont totalement défoliées (tabl. 4).

Les plantes ainsi mutilées survivent en développant de nouvelles feuilles à partir du méristème primaire (Rosette centrale) et des méristèmes secondaires (Rosettes axillaires). La défoliation est suivie d'une mise à fleurs pour certaines plantes du lot P7. La floraison n'affecte qu'un nombre limité de plantes du lot P7 et ne se manifeste que si la défoliation a lieu de façon précoce. En revanche aucune floraison ne s'observe après défoliation pour le lot L3-2 1A ; de même il n'y a aucune montaison dans le lot témoin P7 II.

\section{DISCUSSION ET CONCLUSION}

Les résultats portant sur le cycle de végétation de la digitale laineuse montrent une très grande variabilité, que ce soit sur le développement végétatif ou sur l'initiation à la floraison. La variabilité observée a été révélée par les conditions de culture tant en milieu contrôlé qu'en milieu naturel. Ceci étant acquis, nous avons pu établir les critères les plus intéressants quant à la sélection ultérieure des variétés les plus favorables à la production. Il s'agit, pendant la phase végétative, de conserver les plantes présentant un développement précoce de rosettes axillaires qui ne déséquilibre pas le développement de la rosette centrale, et d'éliminer les plantes présentant une floraison anticipée dès la première année.

Dans la nature, le cycle biologique de la digitale laineuse se déroule en 18 mois (LOISELEUR, 1980). En conditions contrôlées ce cycle a été ramené à 7 mois (fig. 6), avec une phase végétative de 14 semaines et une phase florale de 13 semaines. Ainsi, la connaissance de ce cycle constitue un outil de base : il permet en sélection de tester différents croisements possibles et d'obtenir du matériel à différents stades morphologiques pour la multiplication végétative ; en laboratoire, il permet d'étudier, par exemple, l'évolution de la teneur en digoxine en relation avec le développement.

Au cours de la phase végétative, nous avons montré que l'apparition des rosettes axillaires est liée à la levée de la dominance apicale, le méristème apical de la 
TABLEAU 3

Influence de la valeur de l'éclairement sur l'expression florale pour le lot L3-2 IA.

Conditions de culture : - Prévernalisation, $9 \mathrm{~h}, 22{ }^{\circ} \mathrm{C}, 300 \mu \mathrm{Em}^{-2} \mathrm{~s}^{-1} ;-$ Vernalisation, $9 \mathrm{~h}, 22{ }^{\circ} \mathrm{C}, 250 \mu \mathrm{Em}^{-2} \mathrm{~s}^{-1} ;-\mathrm{Post}_{\text {-vernalisation, }}$ $9 \mathrm{~h}, 22^{\circ} \mathrm{C}$, éclairement naturel ou éclairement artificiel.

$a b c:-a:$ nombre de plantes en montaison $;-b:$ délai en jours entre la fin du froid et le début de la montaison ; $-c:$ HP hampe principale, HAX hampe axillaire, $(f)$ hampe axillaire fine, $(t)$ hampe axillaire à développement tardif, (s) hampe axillaire à développement simultané avec $H P$.

Nombre de plantes par condition : 5 .

Influence of light intensity on flower expression in sample L3-2 IA.

Growth conditions : - before vernalization, $9 \mathrm{~h}, 22^{\circ} \mathrm{C}, 300 \mu \mathrm{Em}^{-2} \mathrm{~s}^{-1}$; - vernalization, $9 \mathrm{~h}, 22{ }^{\circ} \mathrm{C}, 250 \mu \mathrm{Em}^{-2} \mathrm{~s}^{-1}$; - after vernalization, $9 \mathrm{~h}, 22{ }^{\circ} \mathrm{C}$, natural light or artificial light.

$a b c:-a:$ number of bolting plants; $-b:$ time in days between end of cold treatment and beginning of bolting ; $-c:$ HP main stem, $H A X$ axillary stem, $(f)$ fine axillary stem, $(t)$ late developed axillary stem, $(s)$ axillary stem developed simultaneously with $H P$.

Number of plants per condition : 5 .

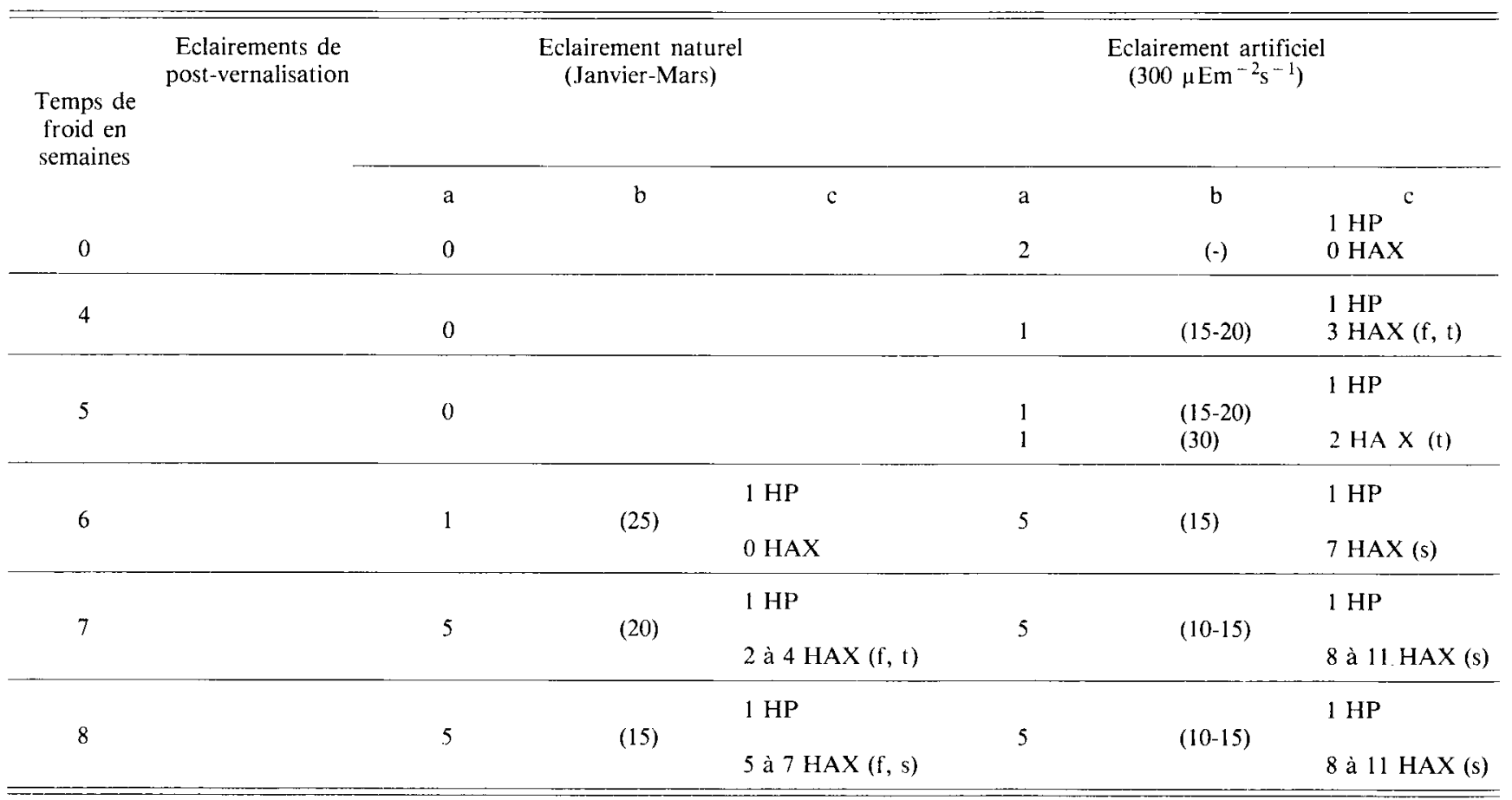

TABLEAU 4

Influence de défoliations pratiquées à des stades morphologiques différents sur la mise à fleurs.

Conditions de croissance : repiquage champ, stade 5-10 feuilles le 7.5.85.

$a b:-a:$ nombre de plantes en montaison; $-b:$ délai en jours entre la défoliation et le début de la montaison. Nombre de plantes traitées : 10

Influence of defoliation at various morphological stages on flowering.

Growth conditions : bedding plants in field ; 5-10-leaf stage.

$a b:-a:$ number of bolting plants $;-b:$ time in days between defoliation and beginning of bolting. Number of plants treated : 10.

\begin{tabular}{|c|c|c|c|c|c|c|c|c|c|c|c|c|}
\hline \multirow[b]{2}{*}{$\begin{array}{c}\text { Lots } \\
\text { L3-2 1A }\end{array}$} & \multirow[t]{2}{*}{$\begin{array}{l}\text { Date de } \\
\text { défoliation }\end{array}$} & \multicolumn{2}{|c|}{$(26.6 .85)$} & \multicolumn{2}{|c|}{$(10.7 .85)$} & \multicolumn{2}{|c|}{$(24.7 .85)$} & \multicolumn{2}{|c|}{$(4.9 .85)$} & \multicolumn{2}{|c|}{$(24.9 .85)$} & \multirow{2}{*}{ 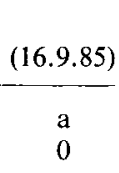 } \\
\hline & & $\begin{array}{l}\mathrm{a} \\
0\end{array}$ & $b$ & $\begin{array}{l}\mathrm{a} \\
0\end{array}$ & $\mathrm{~b}$ & $\begin{array}{l}\mathrm{a} \\
0\end{array}$ & b & $\begin{array}{l}a \\
0\end{array}$ & b & $\begin{array}{l}\mathrm{a} \\
0\end{array}$ & b & \\
\hline $\mathrm{P} 7$ & & 2 & $\begin{array}{l}\text { (15) } \\
\text { (35) }\end{array}$ & 3 & (20) & 2 & $\begin{array}{l}\text { (15) } \\
(20)\end{array}$ & 0 & & 0 & & 0 \\
\hline $\begin{array}{l}\text { P7 } \\
\text { (non défolié) }\end{array}$ & & 0 & & 0 & & 0 & & 0 & & 0 & & 0 \\
\hline
\end{tabular}




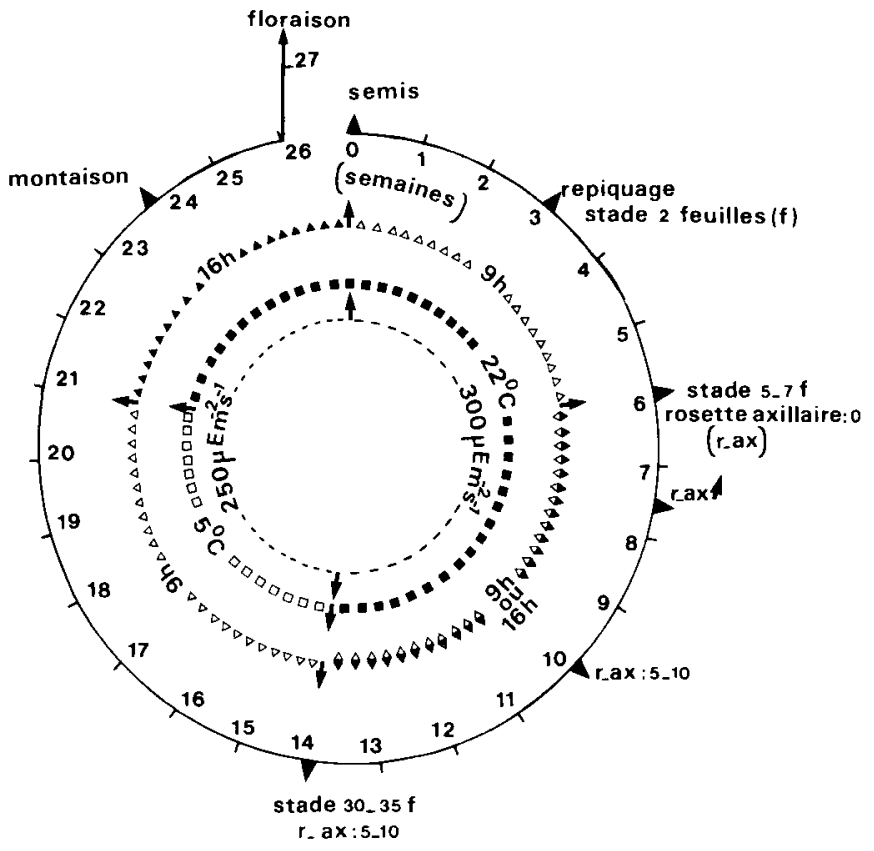

Figure 6

Exemple d'un cycle de végétation de la digitale laineuse en conditions contrôlées (7 mois).

Example of the vegetative cycle of Digitalis lanata under controlled conditions (7 months).

rosette centrale inhibant les bourgeons axillaires. La valeur, la durée et la qualité spectrale de l'éclairement sont des effecteurs positifs pour lever cette dominance apicale. D'un point de vue pratique l'éclairement de type "Grolux Sylvania» de $300 \mu \mathrm{Em}^{-2} \mathrm{~s}^{-1}$, que ce soit en $16 \mathrm{~h}$ ou en $9 \mathrm{~h}$ assure un bon fonctionnement de la rosette centrale et des rosettes axillaires. Toutefois, même sous cet éclairement particulièrement favorable au développement végétatif, des différences sont décelées entre les divers lots de plantes P7, P9, L3-2 et L4-2 dans la vitesse d'émergence des rosettes axillaires. L'éclairement de type «Blanc Industrie » de $250 \mu \mathrm{Em}^{-2} \mathrm{~s}^{-1}$, fournit un moyen de détecter les plantes ayant une faible propension à former des rosettes axillaires. En effet en $16 \mathrm{~h}$ le développement de rosettes axillaires de plantes L3-2 1A est général, tandis qu'en $9 \mathrm{~h}$ seules quelques plantes conservent la capacité de former des rosettes axillaires. L'absence d'expérimentation sous un éclairement de $16 \mathrm{~h}$ de nature strictement photopériodique $(9 \mathrm{~h}$ d'éclairement trophique et $7 \mathrm{~h}$ d'éclairement faible sans effet sur la photosynthèse) ne nous permet pas de conclure que l'action d'un éclairement de 16 h est de nature typiquement photopériodique. Il est manifeste que si ce type d'action ne peut être exclu, l'éclairement agit par l'intermédiaire de son action trophique sur le développement des rosettes axillaires du $D$. lanata.

En effet, sous un éclairement intense et riche en radiations photosynthétiquement actives ( Grolux Sylvania », de $300 \mu \mathrm{Em}^{-2} \mathrm{~S}^{-1}$ ), le développement des rosettes axillaires est stimulé en $9 \mathrm{~h}$ et en $16 \mathrm{~h}$ et s'explique par une stimulation de la production de glucides. La faible stimulation de rosettes axillaires en $9 \mathrm{~h}$ sous un éclairement «Blanc Industrie» peut être la consé- quence de la pauvreté relative de cet éclairement en radiations rouges.

De la même façon Mc INTYRE (1969) attribue la levée de la dominance apicale à une modification trophique ; une augmentation de la production de glucides favoriserait le développement des rosettes axillaires. Par ailleurs, plusieurs auteurs ont décrit l'effet stimulateur du rouge, via le phytochrome (KHUDAIRI et al., 1971 ; LETOUZE, 1972) sur le développement des bourgeons axillaires. Néanmoins, l'effet de l'environnement sur la levée de la dominance apicale est un phénomène plus complexe, car il peut modifier l'équilibre hormonal (MIGINIAC, 1974).

Les expériences sous les divers éclairements n'ayant pas eu lieu avec le même matériel végétal permettent seulement de conclure que, pour des lots de plantes analogues au lot L3-2 1A, l'éclairement « Blanc Industrie » en $9 \mathrm{~h}$ assurera une discrimination entre plantes de différentes capacités à former des rosettes axillaires.

Sous $250 \mu \mathrm{Em}^{-2} \mathrm{~s}^{-1}$ en jours longs, nous avons montré que l'inhibition induite par la dominance apicale dépend du stade de développement, les plantes d'une vingtaine de feuilles étant les plus aptes à développer des rosettes axillaires. De même, ALI \& FLETCHER (1970) avaient mis en évidence un résultat semblable sur les bourgeons axillaires du haricot. Nos expériences ont de plus montré que la précocité de développement et le nombre des rosettes axillaires dépend aussi de l'intensité de la dominance apicale propre à chaque plante.

En conditions naturelles sur des lots de 60 plantes (résultats non montrés) nous avons pu relier la variabilité phénotypique observée (précocité de développement et nombre des rosettes axillaires) à des facteurs externes (notamment la photopériode et l'intensité lumineuse) et à des facteurs internes liés au génotype de la plante. Dans le but d'obtenir un développement homogène et d'augmenter le rendement à l'hectare, la sélection, sur le terrain, portera sur les plantes les plus vigoureuses qui assurent un développement équilibré entre la rosette centrale et les rosettes axillaires. Les plantes qui génétiquement sont les moins sensibles à l'action des facteurs externes pour l'initiation des rosettes axillaires seront donc sélectionnées.

L'étude du développement reproducteur a été engagée afin de repérer des plantes bisannuelles strictes et ainsi d'éviter la montaison durant la première année. Nous avons tout d'abord étudié l'effet inducteur du froid comme c'est le cas dans la nature. Au stade 3035 feuilles, après 7 semaines de froid, toutes les plantes fleurissent. Nous avons également défini la durée de vernalisation nécessaire à la floraison de plantes plus jeunes (stade 20-25 feuilles, 10 semaines de froid). Les résultats obtenus sont typiques de plantes présentant un besoin de vernalisation (MARGARA, 1960 ; PICARD, 1968). 11 est probable, que le choix des conditions expérimentales (température, photopériode, éclairement) avant, pendant et après la vernalisation est essentiel. En effet, ces conditions peuvent modifier le stade de développement sensible au froid ainsi que la durée du froid, comme cela se produit chez Oenothera biennis L. (PICARD, 1968).

Enfin, la vernalisation est un moyen de différencier les lots quant à leur aptitude à la floraison. Nous avons montré que le lot $\mathrm{P} 7$ est plus « sensible » à la vernali- 
sation que le lot $\mathrm{P} 9$, ce dernier présentant des plantes avec une phase juvénile plus longue et une réponse florale plus homogène.

Pour l'expression florale de la digitale laineuse, il semble exister, selon les plantes, des différences de sensibilité vis-à-vis de la photopériode. La floraison après induction par le froid n'est possible, pour toutes les plantes étudiées, qu'en jours longs. Ce résultat diffère de celui obtenu par SIDOROVA (cité par YANIV, 1985), qui décrit cette espèce plutôt comme une plante indifférente.

Le froid n'est pas le seul facteur inducteur de la floraison; nous avons établi que l'éclairement de $300 \mu \mathrm{Em}^{-2} \mathrm{~s}^{-1}$ peut également être stimulateur. L'influence de cet éclairement et l'action synergique froid-éclairement sur la mise à fleurs ont été observées chez les plantes du lot L3-2 1A. De la même façon, un éclairement intense associé à une bonne nutrition minérale provoque une croissance vigoureuse, puis la mise à fleurs sans vernalisation chez le Geum urbanum L. (CHOUARD \& TRAN THANH VAN, 1964). Un effet synergique du froid et de l'éclairement a également été mis en évidence chez le Dactylis glomerata L. (BLONDON, 1972).

L'éclairement d'intensité élevée fourni isolément ou en association avec une période de froid est donc un moyen supplémentaire pour faire apparaître au sein d'une population, les individus sensibles à la montaison.

Ainsi, en conditions contrôlées, 2 niveaux de sélection peuvent être envisagés en utilisant séparément ou en association les 2 traitements suivants :

- obtention d'un lot d'individus les moins sensibles à la montaison. La culture sous un éclairement de $300 \mu \mathrm{Em}^{-2} \mathrm{~s}^{-1}$ permet d'éliminer les plantes les plus sensibles ;

- obtention d'un lot le plus exigeant quant à la mise à fleurs. Soumettre des plantes à des temps de vernalisation variables permettra de déterminer les différents stades de maturité et les durées de froid nécessaires à leur floraison. Ainsi, des degrés de résistances propres à chaque lot seront révélés.

En conditions naturelles où le contrôle des facteurs externes est impossible, nous proposons une expérimentation basée sur la défoliation. Nos résultats montrent que la défoliation pratiquée à un stade jeune permet de séparer 2 groupes de plantes répondant différemment à la montaison. Ce phénomène est à rapprocher de l'inhibition de l'induction florale exercée par les feuilles adultes chez d'autres plantes en rosettes à l'état végétatif comme les Blitum sp. (JACQUES, 1977) ou le fraisier (THOMPSON \& GUTTRIDGE, 1960). Il reste à déterminer si effectivement dans ces conditions naturelles nous sélectionnons par la défoliation le même type de plantes que celles sélectionnées en conditions contrôlées. Une étude de la vernalisation en conditions contrôlées à partir des plantes sélectionnées au champ pourrait être envisagée.

Le présent travail apporte quelques éléments pour sélectionner la digitale laineuse sur des exigences de production : il s'agit ici plus spécialement d'une augmentation du rendement de matière sèche par surface de culture. Cependant, il faut garder à l'esprit qu'une augmentation de la masse foliaire n'est pas obligatoirement corrélée à une augmentation parallèle de la teneur en digoxine. A la notion de l'exigence de quantité de matière première doit s'ajouter la notion de « qualité ». Il s'agira alors de sélectionner les plantes à hautes teneurs en digoxine.

Ensuite sur les clones obtenus par multiplication végétative des plantes sélectionnées, il deviendra possible de préciser le cycle biologique de $D$. lanata et d'affiner les éléments de sélection, dégagés ici à partir d'un matériel varié.

Reçu le 26 janvier 1987. Accepté le 6 juillet 1987.

\section{RÉFÉRENCES BIBLIOGRAPHIQUES}

Ali A., Fletcher R. A., 1970. Hormonal regulation of apical dominance in soybeans. Can. J. Bot., 48, 1989-1994.

Blondon F., 1972. Facteurs externes et déterminisme floral d'un clone de Dactylis glomerata L. In : Phytotronique et Prospective Horticole. Gauthier-Villars éd., Paris, 135-181.

Chouard P., 1969. Croissance, développement, reproduction, sénescence. In : Physiologie de la Croissance à la Sénescence des Végétaux. Encyclopédie de la Pléiade, 1159-1173.

Chouard P., Tran Thanh Van M., 1964. Vernalisation sans réfrigération par les facteurs trophiques de la vigueur, grande luminosité et niveau élevé de la nutrition minérale, chez le Geum urbanum. C. $R$. Acad. Sc., Sér. D, Paris, 259, 4783-4784.

De Bilderling N., Lourtioux A., 1976. Quinze années de phytotronique. In : Etudes de Biologie Végétale. Hommage au Professeur Pierre Chouard, R. Jacques éd., Paris, 331-341.

Herrisset A., 1968. La digitale laineuse. Plantes médicinales et phytothérapie, 2, 75-80.

Hollman A., 1985. Plants and cardiac glycosides. Br. Heart J., 54, 258-261.

Jacques M., 1971. Modalités d'action des différents procédés de l'induction florale chez 2 Chénopodiacées de jour long: le Blitum capitatum L. et le Blitum virgatum L. Physiol. Vég., 9, 461-474.
Khudairi A. K., Johnnykutty A. T., Agarwall S., 1971. Phytochromemediated bud development in Pisum sativum. Planta, 101, 185-188. Letouzé R., 1972. Phytochrome et croissance du bourgeon axillaire chez Salix babylonica L. C. R. Acad. Sc., sér. D, Paris, 275, 2663 2666.

Lang A., 1965. Physiology of flower initiation. In : Encyclopedia of Plant Physiology, W. Ruhland ed, Springer-Verlag, Berlin, 15, 1479-1509.

Levy G., 1981. Contribution à l'amélioration du taux d'hétérosides chez Digitalis lanata Ehrh. Mémoire D.E.A., Université de Clermont II, $29 \mathrm{p}$

Loiseleur J. M., 1980. La production de la Digitale laineuse dans le département du Maine-et-Loire. Institut Supérieur Industriel de l'Etat, Huy-Gembloux, Verviers, section Agriculture, 148 p.

Mc Intyre G. I., 1969. Apical dominance in the rhizome of Agropyron repens. Evidence of competition for carbohydrates as a factor in the mechanism of inhibition. Can. J. Bot., 47, 1189-1197.

Margara J., 1960. Recherches sur le déterminisme de l'élongation et de la floraison dans le genre Beta. Thèse Doct. Etat, Paris, 117 p.

Mastenbroek C., 1979. Some experiences in Digitalis lanata. Herba Hungarica, 18, 167-173.

Mastenbroek C., 1985. Cultivation and breeding of Digitalis lanata in the Netherlands. Br. Heart J., 54, 262-268. 
Miginiac E., 1974. Quelques aspects morphologiques, physiologiques et biochimiques de la dominance apicale. Physiol. Vég., 12, 689-670.

Miginiac E., 1978. Some aspects of regulation : role of correlative factors in photoperiodic plants. Bot. Mag. Tokyo Special Issue, 1, 159-173.

Paris R. R., Moyse H., 1971. Digitalis lanata Ehrh. In : Matière Médicale, collection "Précis de pharmacie », Masson éd., Paris,

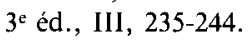

Pasquier B., 1983. Contribution à l'étude de la biologie florale de Digitalis lanata Ehrh., Mémoire D.E.A., Université de Clermont II, $49 \mathrm{p}$.

Phelouzat R., 1970. La phase inflorescentielle de la Digitale pourpre : Digitalis purpurea L. C. R. Acad. Sc., sér. D, Paris, 270, 29272928.
Phelouzat R., Picard C., 1968. Aspects histologique et physiologique de la floraison des bourgeons axillaires et du bourgeon terminal chez la Digitalis purpurea L. C. R. Acad. Sc., sér. D, Paris, 267, 2151-2154.

Picard C., 1966. Contribution à la connaissance de la vernalisation de Ia Digitale pourpre, Digitalis purpurea L. C. R. Acad. Sc., sér. D, Paris, 262, 95-97.

Picard C., 1968. Aspects et mécanismes de la vernalisation. Collection de monographie de Botanique et de Biologie Végétale, Masson éd., Paris, $128 \mathrm{p}$.

Thompson P. A., Guttridge C. G., 1960. The role of leaves as inhibitors of flower induction in strawberry. Ann. Bot., N.S., 24, 482-490.

Yaniv Z., 1985. Digitalis. In : Handbook of Flowering, 2, CRC Press, Boca Raton, Fl., 7, 446-449. 